\title{
Peer review report 2 on "Dew as an Adaptation Measure to Meet Water Demand in Agriculture and Reforestation"
}

$\mathrm{PhD}$, Associate Professor

Wenceslao González-Viñas

University of Navarra, Physics and Applied Mathematics

c/Irunlarrea 1, Pamplona, E-31008,

Spain

\section{Original Submission}

\subsection{Recommendation}

Major Revision

\section{Comments to Author:}

The authors analyze the possible use of dew water as a source of plant irrigation, specially in arid/semi-arid regions, or other that are prone to droughts. They compare and discuss dew yields in Beiteddine (Lebanon) with regard to the required amount of water by the considered crops and trees seedlings in order to grow properly. Although this is a very interesting work to the field there are a number of issues that have to be taken into account and fixed accordingly by the authors, prior to be published in "Agricultural and Forest Meteorology".

Some of them are the following:

1) The authors should consider to extend their study to more than one dry season (i.e. to more than a year) because this would have several benefits:

a) It would provide a much better statistics. Conclusions about the usefulness about dew harvesting (which I don't doubt) in this experimental conditions will be much more robust, and convincing to reforestation organizations and agriculture ministries.

b) If the temporal series are long enough, they would give a hint of the effect of climate change in the use of dew harvesting. In that sense, it would be very interesting to know whether dew harvesting in these regions would be every time more useful or less. On the other hand, could dew harvesting help to avoid, or delay, climate change?

c) Finally, would dew harvesting in arid/semi-arid areas affect climate? Is there a relevant feedback mechanism? Could we change a semi-arid area to a rainforest (e.g. by promoting growth of tree species that increase dew yield)?

All these are interesting questions, and I know that they are not easy to answer in a conclusive way, but in my opinion it is important to contribute in answering them. Authors can help in this issue either by measuring directly or by using existing experimental data series from others. The former have the strong drawback of delaying too much the publication of this interesting manuscript. The latter would complement their work and it is worth to be included here.

2) Also, it would be nice, for comparison to look for similar results in other regions with different orography or climate profiles (but still arid/semi-arid). I suggest the authors to complement their results with such an analysis. 
3) Dew is different from fog. Both may be harvested depending (e.g.) on the relative orientation of "dew condensers" to the wind. It would be interesting to report at least the dew/fog yield ratio to better characterize how these mechanisms are involved.

4) Soil type seems to be important to the wilting point and field capacity, and surely it is related to the crops which will grow better. The effect of soil to the dew condensation should be also explored or, at least, discussed.

5) Along most of the manuscript, data lack (statistical) standard error. At least, an estimation would be needed to do some statistical inference about usefulness.

Other minor issues that also should be taken into account are:

7) (rows 17-18) The ('significant') impact of dew on diurnal soil moisture is without condensers or with condensers and using the harvested dew to water the plants or what ...? It should be clear as it is a highlight (3rd)!

8) (rows 37-39) Related to the previous comment. It is confusing . . . Does dew significantly' affect or not diurnal soil moisture Also, how is usually the absorption performed? In a chemical way (thus, implying a chemical reaction)? In the cases where there is no such chemical reaction, what is the difference between dew and water vapor absorption?

9) (row 57) Abbreviation "msl" should be written in full (except the part which is consistent with the international system of units).

10) (row 142) 'area of $2 \mathrm{~m}^{2}$ ' In other places were $1 \mathrm{~m}^{2}$. It should be properly distinguished from the beginning where/when 1 or 2 was used, as otherwise it is confusing.

11) (rows 162-163) Here it is stated that the evaporation occurs at maximum rate when the soil surface is wet. However, this would depend also on the \%RH in each case. . Please, comment on this.

12) (rows 270-272) I think that this sentence is very relevant to real world applications and, in some way, should be included in the abstract. Could it be a highlight itself?

13) (caption of Table 1) It should give what are the columns RH, Wind and Rain? I assume they are averages...

All in all, this is a very promising work and it would merit publication after my issues above are considered.

\section{First revision}

\subsection{Recommendation}

Minor Revision

\section{Comments to the author}

Almost all my recommendations have been addressed by the authors in the revised manuscript. After consideration of the following optional remark, the manuscript can be published:

With regard to my former comment 3. Although from a technology point of view, I concur with the authors that fog and dew harvesting are well different in order to optimize the reached yield. However, I think that in both techniques, both phenomena occur (fog and dew) and sometimes even in the scientific literature there is some confusion between the two. Please, have a look into J. Guadarrama-Cetina et al., "Dew condensation on desert beetle skin" Eur. Phys. J. E (2014) 37: 109 (DOI 10.1140/epje/i2014-14109-y) where it is clearly stated (and proved) that this confusion happened in a number of previously published papers. 